\title{
Anxiety and the worry process
}

\author{
MICHAEL W. EYSENCK \\ Birkbeck College, University of London, London, England
}

\begin{abstract}
Subjects who were either high or low in trait anxiety received mood-induction procedures designed to produce either an anxious or a nonanxious mood. The amount of worrying that these subjects engaged in when cued with one of their current worries was greater among those high in trait anxiety than among those low in trait anxiety. In addition, anxious mood induction produced more worrying initially than did nonanxious mood induction. In general terms, the findings indicated that anxious induced mood can facilitate the initiation of the worry process, but its subsequent maintenance is far more a function of trait anxiety. It was concluded that prolonged worry occurs mainly in those individuals who have highly organized clusters of worry-related information stored in long-term memory.
\end{abstract}

In view of the obvious importance of worrying as a psychological phenomenon and clinical problem, it is rather surprising that there has been relatively little systematic investigation of the factors that produce and sustain worry. The approach taken here was to examine in some detail possible relationships between worry and anxiety. One possibility is suggested by research on state-dependent effects (Bower, 1981). In essence, memories that are acquired when an individual is in a particular emotional state or mood are most readily recalled subsequently when that person is in the same, or a similar, mood. In terms of worry, the implication is that the anxiety-related information typically incorporated into the worry process will be most accessible when an individual is already anxious, and thus the presence of an anxious mood will increase both the probability of occurrence of worry and the duration of the worry process. If, in addition, the retrieval of anxiety-related information from long-term memory leads to an increase in feelings of anxiety, then a vicious circle may develop, in which anxious mood facilitates retrieval of anxiety-related information, and retrieval of such information increases anxiety.

A further possibility (not necessarily incompatible with the one just discussed) is that dispositional characteristics (e.g., trait anxiety) are importantly related to worry. Individuals who are high in trait anxiety may have wellorganized collections of anxiety-related information stored in long-term memory. For example, in the case of anxiety patients, Butler and Mathews (1983) obtained evidence for the existence of "danger schemata," that is, cognitive structures in memory relating to threat evaluation. If individuals high in trait anxiety possess many more anxiety-related schemata than individuals low in trait anxiety, then this could account for individual differences in the frequency and duration of worry (cf. Borkovec, Robinson, Pruzinsky, \& DePress, 1983).

The author's mailing address is: Department of Psychology, Birkbeck College, University of London, Malet Street, London, WCIE 7HX, England.
There is very little evidence in the literature concerning the relative importance of transient anxious mood and semipermanent trait anxiety in affecting worry. However, a partially relevant study was reported by Mayo (1983). He asked subjects to recall specific real-life personal experiences from memory when provided with stimulus words. The personality dimension of neuroticism (which is highly correlated with trait anxiety) was related to retrieval of more unpleasant and unhappy memories, whereas the subjects' moods were almost unrelated to the pleasantness or unpleasantness of the retrieved memories.

For present purposes, the study by Mayo (1983) suffered from two major limitations. First, he considered only the first memory retrieved to each stimulus word, whereas worry research focuses on the nature of the information retrieved over relatively long periods of time. Second, Mayo did not manipulate subjects' mood states. As a consequence, the failure of mood to affect retrieval may be attributable simply to the nonextreme moods experienced by most of the subjects.

In sum, the present study was designed to investigate several aspects of the relationship between anxiety and worry. More specifically, the respective roles of anxious mood and trait anxiety in affecting worry were of interest, as was the impact of worry itself on experienced mood. In order to provide maximal information on these issues, the time course of the worry process was carefully monitored.

\section{METHOD}

\section{Design}

The experimental session comprised two major parts. The subjects received an anxious (or nonanxious) mood induction in the first part of the experiment, followed by a 5 -min period of thinking time. In the second part of the experiment, the opposite mood was induced, and was followed by a further 5-min period of thinking time. The presence or absence of worry was assessed every 15 sec during both periods of thinking time. The order of mood induction was random, with half of the subjects receiving one order, and the other half the other order.

The subjects first completed the trait measure of the State Trait Anxiety Inventory (STAI) (Spielberger, Gorsuch, \& Lushene, 1970). Then they 
thought of two current worries, followed by the presentation of the instructions. Next, the first state measure of the STAI was completed. Mood induction was followed immediately by the mood measure and thinking period. Directly after the thinking period, the second mood measure was completed, and the subjects estimated the percentage of the thinking period they had spent worrying and completed the second state measure of the STAI. After a 7-min rest, the instructions were briefly recapitulated, and the second part of the experiment followed along the lines of the first part of the experiment, but with the other mood being induced and the thinking period being based on the other worry.

\section{Subjects}

There were 44 subjects who were all students at the University of London. They were selected from an initial pool of 60 subjects on the basis of having relatively extreme scores on the trait measure of the STAI. They were all between the ages of 18 and 35 years and received modest payment for their participation in the study.

\section{Mood Induction}

The method of mood induction was broadly similar to that described by Velten (1968). For both anxious and nonanxious mood induction, the subjects read 12 cards bearing typed self-referent statements. The statements were carefully selected to ensure that they did not refer to aspects of the subjects' actual experiences. Sample statements are "I feel very insecure" (anxious mood) and "I feel relaxed and at ease" (nonanxious mood).

The measure of mood was based on the one used by Teasdale and Taylor (1981). Anxious mood was rated using a line scale $10 \mathrm{~cm}$ long, the left end of which was labeled "I do not feel at all anxious" and the right end of which was labeled "I feel extremely anxious." In order to make it clear that current mood was to be rated, the phrase "AT THIS MOMENT" was written above the line. Ratings were made by means of a vertical line cutting through the mood scale.

\section{Procedure}

Upon their arrival, the subjects were asked to complete the trait measure of the STAI. Next, they were asked to think of two current worries; these worries were to be of moderate intensity, and were to be of comparable intensity. The subjects provided a brief description of each worry and rated its intensity on a 100-point scale ranging from 0 ("not worrying at all") to 100 ("the most worrying thing you can possibly imagine"). Only worries with ratings between 40 and 70 were accepted; any subjects who mentioned worries outside this range were asked to think of further moderate-intensity worries. Four subjects could not think of two worries inside the designated range, and their data were discarded.

The subjects were then provided with instructions for the first part of the experiment. The necessity of doing their best to feel the mood suggested by the statements in the mood-induction procedure was emphasized. They were told to try to avoid thinking about actual events from their own lives while being presented with the cards; rather, they were to attempt to enter into the desired mood state as directly as possible by concentrating on the statements. The induction procedure would last for $6 \mathrm{~min}$, because each statement would be presented for $15 \mathrm{sec}$, and the whole pack of statements would then be re-presented for a further $15 \mathrm{sec}$ each.
The instructions for the thinking period indicated that the subjects would be presented with brief descriptions of one of their worries. One of the two worries was to be selected at random. They were to try to think about the source of that worry and to see whether they naturally kept worrying about it during the following $5 \mathrm{~min}$. An auditory stimulus would be presented every $15 \mathrm{sec}$ during this period. Upon hearing this signal, the subjects were to say "Yes" if they had been worrying when the signal occurred, "No" if they had not, and "Maybe" if they were uncertain. Occasionally, the experimenter would say, "Describe," after a signal occurred, which indicated that the subjects were to describe briefly their thoughts at the time of the signal. The subjects were actually asked to describe their thoughts after the 10th and 20th signals.

\section{RESULTS AND ANALYSIS}

Each subject indicated at 20 points within each thinking period whether he or she was currently worrying. A score of " 2 " was assigned to each "Yes" response and of " 1 " to each "Maybe" response. .Two independent judges read the descriptions of the thoughts provided by the subjects after the 10th and 20th signals, and assigned them to the "Yes," "Maybe," and "No" categories. The percentage of categorizations coinciding with those given by the subjects was $91 \%$ for one judge and $88 \%$ for the other judge. The worry data thus appear to possess validity.

For purposes of analysis, the aggregate worry scores for each 75-sec period were calculated, and mean worry scores in the various conditions are shown in Table 1. These worry data were submitted to a three-factor splitplot analysis of variance, with trait anxiety as the betweensubjects factors. Trait anxiety produced a significant effect on worry performance $[F(1,38)=9.57, p<.01]$, with subjects high in trait anxiety worrying for more of the thinking period than those low in trait anxiety. There was a substantial effect of time period $[F(3,114)=11.11$, $\mathrm{p}<.001]$, with the incidence of worrying declining over time. Somewhat surprisingly, there was no effect of induced mood on the amount of worry activity $(F<1)$.

However, the above main effects need to be qualified. There was a significant interaction between induced mood and time period $[\mathrm{F}(3,114)=9.20, \mathrm{p}<.001]$. Anxious induced mood produced more worry than nonanxious induced mood during the first time period $[\mathrm{F}(1,152)=5.21$, $\mathrm{p}<.05$ ], but the opposite was the case during the third and fourth time periods. There was also a three-way interaction of trait anxiety, induced mood, and time period $[F(3,114)=5.02, p<.01]$. The meaning of this interaction was ascertained by considering some of the sim-

Table 1

Worry Scores (WS) and Standard Deviations (SD) as a Function of Trait Anxiety, Induced Mood, and Time Period

\begin{tabular}{|c|c|c|c|c|c|c|c|c|c|c|c|c|c|c|c|}
\hline \multicolumn{8}{|c|}{ Low Anxious Mood } & \multicolumn{8}{|c|}{ High Anxious Mood } \\
\hline \multicolumn{2}{|c|}{1} & \multicolumn{2}{|c|}{2} & \multicolumn{2}{|c|}{3} & \multicolumn{2}{|c|}{4} & \multicolumn{2}{|c|}{1} & \multicolumn{2}{|c|}{2} & \multicolumn{2}{|c|}{3} & \multicolumn{2}{|c|}{4} \\
\hline WS & SD & WS & SD & WS & $\mathrm{SD}$ & WS & SD & WS & SD & WS & SD & WS & SD & WS & SD \\
\hline \multicolumn{16}{|c|}{ High Trait Anxiety } \\
\hline 6.35 & 2.68 & 6.20 & 2.65 & 5.95 & 2.46 & 5.60 & 3.14 & 6.70 & 2.34 & 6.60 & 2.98 & 5.80 & 1.92 & 5.95 & 1.70 \\
\hline \multicolumn{16}{|c|}{ Low Trait Anxiety } \\
\hline 4.45 & 2.44 & 4.75 & 3.04 & 4.40 & 2.60 & 3.80 & 2.93 & 6.25 & 2.73 & 4.90 & 2.27 & 3.80 & 2.29 & 2.80 & 1.85 \\
\hline
\end{tabular}


ple main effects. There was no effect of time period among subjects high in trait anxiety, whether they were in anxious induced mood $[\mathrm{F}(3,228)=1.96]$ or in nonanxious induced mood $[\mathrm{F}(3,228)=1.02]$, or among subjects low in trait anxiety in nonanxious induced mood $[F(3,228)=$ 1.51]. However, there was a substantial effect of time period on worry performance among subjects low in trait anxiety in anxious induced mood $[\mathrm{F}(3,228)=20.92$, $\mathrm{p}$ $<.001]$. Thus, it was only those low in trait anxiety whose worry scores were affected by the mood-induction procedure, and worry declined significantly over time only among those low in trait anxiety who were exposed to the anxious mood induction.

It was possible to obtain independent evidence concerning some of the above effects on worry by analyzing the data on the basis of the percentage of time that the subjects reported they had spent worrying. The summary data are shown in Table 2. A two-factor split-plot analysis of variance was carried out on these data, with trait anxiety as the between-subjects factor and induced mood as the within-subjects factor. Trait anxiety had a highly significant effect on worry $[F(1,38)=11.79, p<.01]$, with subjects high in trait anxiety reporting a much higher percentage of time spent worrying than subjects low in trait anxiety. There was no significant main effect of induced $\operatorname{mood}(\mathrm{F}<1)$, and the interaction between trait anxiety and induced mood was also nonsignificant $(F<1)$. Thus, the pattern of results based on the worry-percentage data closely resembled that based on immediate reporting of the presence or absence of worrying in response to the auditory signals.

The measure of anxious mood taken just prior to the thinking period and immediately afterwards provides an indication of the success or otherwise of the moodinduction procedure, and of the effects of worry on anxious mood. The summary mood data are given in Table 3. These data were submitted to a three-way split-plot analysis of variance, with trait anxiety as the between-subjects factor and induced mood and temporal position (i.e., before vs. after the thinking period) as the within-subjects factors. There was a highly significant main effect of trait anxiety on anxious $\operatorname{mood}[\mathrm{F}(1,38)=24.70, \mathrm{p}<.001]$, and the mood-induction procedure had its expected effect on anxious mood $[\mathrm{F}(1,38)=29.70, \mathrm{p}<.001]$. Mood was more anxious before the thinking period than afterwards $[\mathrm{F}(1,38)=18.31, \mathrm{p}<.001]$.

The interpretation of these main effects is affected substantially by two large interaction effects. There was a

Table 2

Worry Percentage Data (WP) and Standard Deviations (SD) as a Function of Trait Anxiety and Induced Mood

\begin{tabular}{lcccc}
\hline \multicolumn{2}{c}{ Low Anxious Mood } & & \multicolumn{2}{c}{ High Anxious Mood } \\
\cline { 4 - 5 } WP & SD & WP & SD \\
\hline 55.2 & \multicolumn{2}{c}{ High } & Trait Anxiety & \\
& 19.4 & 57.0 & 18.1 \\
37.5 & Low & Trait Anxiety & \\
& 19.4 & 36.2 & 16.9 \\
\hline
\end{tabular}

Table 3

Anxious Mood (AM) and Standard Deviations (SD) as a Function of Trait Anxiety, Induced Mood, and Temporal Position

\begin{tabular}{|c|c|c|c|c|c|c|c|}
\hline \multicolumn{4}{|c|}{ Low Anxious Induced Mood } & \multicolumn{4}{|c|}{ High Anxious Induced Mood } \\
\hline \multicolumn{2}{|c|}{ Start } & \multicolumn{2}{|c|}{ End } & \multicolumn{2}{|c|}{ Start } & \multicolumn{2}{|c|}{ End } \\
\hline $\mathrm{AM}$ & SD & $\mathrm{AM}$ & SD & $\mathrm{AM}$ & SD & $\mathrm{AM}$ & SD \\
\hline \multicolumn{8}{|c|}{ High Trait Anxiety } \\
\hline 46.7 & 16.5 & 47.9 & 19.3 & 61.9 & 14.8 & 51.5 & 20.5 \\
\hline \multicolumn{8}{|c|}{ Low Trait Anxiety } \\
\hline 17.7 & 4.4 & 29.5 & 12.9 & 55.9 & 17.8 & 28.7 & 15.5 \\
\hline
\end{tabular}

Table 4

State Anxiety (SA) and Standard Deviations (SD) as a Function of Trait Anxiety, Induced Mood, and Temporal Position

\begin{tabular}{|c|c|c|c|c|c|c|c|}
\hline \multicolumn{4}{|c|}{ Low Anxious Mood } & \multicolumn{4}{|c|}{ High Anxious Mood } \\
\hline \multicolumn{2}{|c|}{ Start } & \multicolumn{2}{|c|}{ End } & \multicolumn{2}{|c|}{ Start } & \multicolumn{2}{|c|}{ End } \\
\hline SA & SD & SA & SD & SA & SD & SA & SD \\
\hline \multicolumn{8}{|c|}{ High Trait Anxiety } \\
\hline 49.6 & 8.7 & 51.4 & 7.1 & 50.0 & 6.7 & 51.6 & 9.1 \\
\hline \multicolumn{8}{|c|}{ Low Trait Anxiety } \\
\hline 34.6 & 7.1 & 35.2 & 7.9 & 34.5 & 7.1 & 36.9 & 7.2 \\
\hline
\end{tabular}

highly significant interaction between induced mood and temporal position $[F(1,38)=60.56, p<.001]$. In this interaction, there was a very large difference in mood prior to the thinking period as a function of the mood induced, but there was a very much smaller effect of mood induction on mood after the thinking period. In other words, the mood-induction procedure was very successful in altering mood, but the effects were relatively transient.

There was also a significant second-order interaction involving induced mood, temporal position, and trait anxiety $[F(1,38)=17.76, p<.001]$. The general tendency for the effects of mood induction to diminish over time was greatest among subjects low in trait anxiety exposed to anxious mood induction, and was least apparent among high-trait-anxiety subjects exposed to nonanxious mood induction.

Further evidence relevant to the time course of feelings of anxiety during the experiment is available in the state measure of the STAI. Obviously, it was not expected that state anxiety would vary in the same way as anxious mood, because state anxiety was initially assessed prior to mood induction, whereas anxious mood was assessed only after mood induction. The summary data for state anxiety are presented in Table 4 . The state-anxiety data were analyzed by a split-plot analysis of variance incorporating trait anxiety, induced mood, and temporal position as factors. As expected, trait anxiety affected state anxiety $[F(1,38)=61.60, p<.001]$. In addition, there was a small effect of temporal position $[F(1,38)=5.49$, $\mathrm{p}<.05$ ]: State anxiety tended to be greater after the thinking period than before it. There was no effect of induced mood, and none of the interaction effects was statistically significant. 


\section{DISCUSSION}

The findings obtained in this study are relevant to several issues involving the interrelationships of trait anxiety, induced mood, worry, and mood state. It is clear that these factors are related in complex ways that evolve dynamically over time, but this temporal dimension has been ignored in most previous research. One of the advantages of the experimental approach adopted here is that its emphasis on processes operating over time makes it relevant to clinical problems relating to worry.

The value of adopting a temporal perspective can be seen if we consider one of the major issues addressed by this study, namely, the factors that are responsible for the onset and maintenance of worry. In essence, it is possible to distinguish between relatively permanent factors (e.g., predisposition to anxiety as measured by scales of trait anxiety) and more transient factors (e.g., current state or mood). The findings of this study revealed that both factors play a part in the worry process, but trait anxiety was far more consequential than induced mood. Trait anxiety had a substantial effect on the amount of worrying that occurred throughout the thinking period, but induced anxious mood increased worry only during the first $75 \mathrm{sec}$ of the thinking period, and then only for individuals low in trait anxiety. In other words, initiation of the worry process can be facilitated by either high trait anxiety or by anxious induced mood, but prolonged worry occurs largely as a result of high trait anxiety rather than in response to an initial mood.

It may seem fairly obvious that the effects of induced mood on worry would be transient, but there are theoretical and empirical reasons why the effects might have been more prolonged. As Teasdale and his colleagues (e.g., Teasdale \& Taylor, 1981) have argued, there is strong evidence that current mood facilitates the retrieval of information from long-term memory that was acquired in the same or a similar mood, and that retrieval of mood-related information enhances the probability that that mood will be experienced. The combined effects of mood on selectivity of retrieval and of retrieval on mood should theoretically produce a vicious circle. As applied to the specific mood of anxiety and the retrieval of worrying information, the expectation is that anxious induced mood should produce retrieval of worrying information, which then enhances anxious mood, and so on.

Why did this vicious circle fail to develop? It could be argued either that the induced mood was insufficiently intense, or that it was artificially induced rather than naturally occurring, and thus was ineffective. However, although different results might have been obtained with some more powerful manipulation of mood, the technique used here produced large effects on the mood measure. With respect to the artificiality of the induction procedure, it is worth noting that artificially induced moods have previously been found to produce causal effects on performance. Moreover, one would expect mood to be increasingly affected in a more "natural" fashion by the retrieval of worry material as the thinking period progressed.

The preferred interpretation of the data is that prolonged worry occurs primarily in those individuals who have tightly organized clusters of anxiety- or worry-related information stored in long-term memory. The highly structured nature of these "worry clusters" produces two of the more striking characteristics of the worry process: its repetitive nature and its uncontrollability. In other words, the most important determinant of the number and duration of worry episodes experienced by an individual are the number and degree of structure of his or her worry clusters.

If we assume that there are major differences between individuals high and low in trait anxiety in the number and structure of worry clusters they possess, then we have a potential explanation for the key finding that the amount of worry experienced during the thinking period was best predicted by individual differences in trait anxiety. In the case of low-trait-anxiety subjects exposed to anxious mood induction, the induced mood facilitated access to whatever anxiety-related information was stored in long-term memory. However,the relatively small number of worry clusters stored in the long-term memory systems of lowtrait-anxiety subjects shortened the worry process in such subjects. In other words, the factors responsible for sustaining the worry process may be somewhat different from the factors responsible for initiating the worry process. Accordingly, the sizable literature on the immediate effects of mood on retrieval from long-term memory may be relatively uninformative with respect to the more pressing clinical problem of persistent and uncontrollable worrying.

It has already been mentioned that the incidence of worry over time either remained constant or else decreased. This seems at least partially inconsistent with findings reported by Breznitz (1971). He argued that there is an "incubation of threat," with worry increasing progressively over time. However, his experimental evidence was obtained in a very artificial situation, which was also rather unusual in that the genuine cause for concern increased minute by minute.

The emphasis so far has been on the ways in which the worry process is affected by personality and mood. However, it is also possible to consider the effects of worry on mood by comparing anxious mood before and after the thinking period. Mood tended to be less anxious after the thinking period than before it, whereas it might have been expected that worry would increase feelings of anxiety. The group of subjects showing the greatest reduction in anxious mood during the thinking period consisted of low-trait-anxiety subjects given the anxious mood induction. Since the same subjects were the only ones whose worry scores decreased during the thinking period, there is an interesting parallelism between the time courses of their mood and worry scores.

The state-anxiety data suggest that worry may have had some adverse effect on anxiety. It will be remembered that state anxiety was measured before the mood-induction procedure took place and after the end of the thinking period. Although the effect was rather small, state anxiety was significantly greater after the thinking period than before it. However, this cannot be attributed entirely to the worrying that occurred between test administrations, because the extent of the increase in state anxiety was not responsive to the amount of worrying that occurred.

Returning to the anxious mood data, it is clear that anxious mood at the start of the thinking period is jointly determined by the mood-induction procedure and by trait anxiety. At the end of the thinking period, anxious mood is almost entirely a function of trait anxiety, and the effects of induced mood are negligible. Since trait anxiety was the major determinant of the amount of worrying toward the end of the thinking period, it is possible that the effects of trait anxiety on anxious mood after the thinking period are mediated, at least in part, by the amount of worrying.

In sum, this study represents a preliminary attempt to identify some of the factors responsible for the initiation and maintenance of worry. Although much remains to be discovered, it may be valuable to distinguish between transient factors arising out of the immediate situation and more durable factors such as the information stored in long-term memory.

\section{REFERENCES}

Borkovec, T. D., Robinson, E., Pruzinsky, T., \& DePree, J. A. (1983). Preliminary exploration of worry: Some characteristics and processes. Behaviour Research and Therapy, 21, 9-16.

Bower, G. H. (1981). Mood and memory. American Psychologist, 36, 129-148.

BREZNITZ, S. A. (1971). A study of worrying. British Journal of Social and Clinical Psychology, 10, 271-279.

Butler, G., \& MATHEws, A. (1983). Cognitive processes in anxiety neurosis. Advances in Behaviour Research and Therapy, 5, 51-62.

MaYo, P. R. (1983). Personality traits and the retrieval of positive and negative memories. Personality and Individual Differences, 4, 465-471.

SPielberger, C. D., Gorsuch, R., \& LuSheNE, R. (1970). The State Trait Anxiety Inventory (STAI) test manual. Palo Alto, CA: Consulting Psychologists Press.

Teasdale, J. D., \& TAYlOR, R. (1981). Induced mood and accessibility of memories: An effect of mood state or of induction procedure? British Journal of Clinical Psychology, 20, 39-48.

Velten, E. (1968). A laboratory task for induction of mood states. Behaviour Research and Therapy, 6, 473-482.

(Manuscript received for publication July 26, 1984.) 